M. Hoener, D. Kaczmarek, T. Bierkandt, A. Bodi, P. Hemberger, and T. Kasper, A pressurized flow reactor combustion experiment interfaced with synchrotron double imaging photoelectron photoion coincidence spectroscopy, Rev. Sci. Instrum. 91 (2020) 045115.

The original publication is available at http://scitation.aip.org/content/aip/journal/rsi (C) is with AIP

http://dx.doi.org/10.1063/1.5141168 


\title{
A Pressurized Flow Reactor Combustion Experiment Interfaced with Synchrotron Double Imaging Photoelectron Photoion Coincidence Spectroscopy
}

\author{
M. Hoener ${ }^{1}$, D. Kaczmarek ${ }^{1}$, T. Bierkandt ${ }^{2}$, A. Bodi ${ }^{3}$, P. Hemberger ${ }^{3}$, T. Kasper ${ }^{1}$ \\ ${ }^{1}$ Mass Spectrometry in Reactive Flows - Institute for Combustion and Gas Dynamics (IVG), University \\ Duisburg-Essen, Duisburg 47057, Germany \\ ${ }^{2}$ German Aerospace Center (DLR) - Institute of Combustion Technology, Stuttgart 70569, Germany \\ ${ }^{3}$ Laboratory for Synchrotron Radiation and Femtochemistry - Paul Scherrer Institute, Villigen 5232, \\ Switzerland
}

e-mail for correspondence: martin.hoener@uni-due.de

\begin{abstract}
A new pressurized low-temperature combustion experiment has been commissioned at the Swiss Light Source (SLS), Paul Scherrer Institute. The experiment uses photoionization with tunable synchrotron radiation and double imaging photoelectron photoion coincidence ( $\left.i^{2} \mathrm{PEPICO}\right)$ detection at the vacuum ultraviolet (VUV) beamline. The experimental setup is described including the highpressure reactor experiment, sampling interface, and reactant delivery system. The CRF-PEPICO (Combustion Reactions Followed by Photoelectron Photoion Coincidence) endstation and beamline are briefly elaborated. The novel aspects of the apparatus and the new components are elucidated in detail, such as the fluid supply system to the reactor and the reactor integration into the endstation. We also present a system overview of the experimental setup. The technical details are followed by a description of the experimental procedure used to operate the pressurized flow reactor setup. Finally, first experimental results demonstrating the capability of the setup are provided and analyzed. A major advantage of this new experiment is that the excellent isomer resolution capabilities of the $i^{2} P E P I C O$ technique can be transferred to the investigation of reactions at elevated pressures of several bar. This enables the investigation of the pressure effect on the reactivity of fuel mixtures and to cover more realistic conditions found in technical combustors. The capability to obtain quantitative oxidation data is confirmed and the main and certain intermediate species are quantified for a selected condition. The results show excellent agreement with a chemical kinetics model and previously published reference measurements performed with a gas chromatography setup.
\end{abstract}

\section{INTRODUCTION}

Combustion science investigates interactions of fuels and oxidizers, the kinetics involved in fuel decomposition and oxidation, and the production of pollutants such as soot and NOx. To understand the intricate chemical interactions that take place in combustion processes, universal, highly sensitive and selective detection methods are required. One established technique is photoionization molecular-beam mass spectrometry (PI-MBMS) coupled to a synchrotron vacuum ultraviolet (VUV) photon source (Cool, Mcllroy, et al. (2005); Hansen et al. (2009); Qi (2013); Krüger et al. (2014); Oßwald et al. (2014)). The high photon flux and energy resolution of synchrotron VUV sources make the isomer specific identification of reaction intermediates possible using fragmentation-free soft ionization with high sensitivity and excellent signal to noise ratio (Cool, Mcllroy, et al. (2005); Hansen et al. (2009)). The assignment and, ideally, the accurate concentration measurement of the reaction intermediates in complex gas mixtures at high temperatures and pressures, e.g. in simplified model 
experiments of combustion systems or chemical synthesis processes, are the first steps in the development of reaction mechanisms that describe these complex environments accurately.

The aim of most combustion experiments is to identify and quantify relevant combustion constituents as well as to provide input data and validation for chemical kinetics mechanisms. The advantage of molecular-beam mass spectrometry (MBMS) flame-sampling experiments is that they can shed light on detailed concentration histories of combustion constituents in the flame with high spatial resolution (Hansen et al. (2009); Qi (2013)). Molecular-beam sampling allows for simultaneous identification of all combustion constituents, including that of reactive species, such as radicals, by quenching their destruction reactions by gas beam expansion in a cone-shaped fused silica sampling probe (Hansen et al. (2009); Qi (2013)) before passing them into the analytical stage.

A distinct advantage of gas sampling and species identification using PI-MBMS is the ability to deconvolute isobaric and even isomeric species. This can be achieved by using the energy selective nature of the photoionization process by varying the photon energy of the tunable light source (Cool, Mcllroy, et al. (2005); Hansen et al. (2009); Oßwald et al. (2014); Pieper et al. (2018)). Of particular importance for the development of chemical kinetics mechanisms is the understanding of concentrations and participation of isomeric species in combustion reactions (Krüger et al. (2014); Pieper et al. (2018); Osswald et al. (2007); Cool, Mcllroy, et al. (2005); Hansen et al. (2009)), since they can have significantly different contributions to the reaction network (Cool, Mcllroy, et al. (2005)). The identification of species relies solely on ion yield curves as a function of photon energy (photoionization spectra) in conventional PI-MBMS (Cool, Mcllroy, et al. (2005); Oßwald et al. (2014); Lucassen et al. (2009)). In contrast, the acquired photoelectron spectra using PEPICO allow fingerprinting of species by means of photoion mass selected threshold photoelectron spectra (msTPES) (Oßwald et al. (2014); Pieper et al. (2018); Krüger et al. (2014); Bodi et al. (2013); Hemberger et al. (2013)). These spectra show unique, compound-specific vibronic transitions according to the Franck-Condon (FC) principle (Bodi et al. (2013); Hemberger et al. (2013); Oßwald et al. (2014); Pieper et al. (2018)), which can be compared to both literature references and FC simulation results. Herein lies a particular advantage of photoelectron photoion coincidence spectroscopy (PEPICO) used for combustion diagnostics (Oßwald et al. (2014); Krüger et al. (2014); Pieper et al. (2018)). Identification of species using photoionization (PI) spectra is a powerful tool (Oßwald et al. (2014); Krüger et al. (2014)) and is particularly useful for quantification as well as identification of combustion intermediates in the sub-ppm range. Quantitative data can be obtained through calibration using stable species, literature ionization cross sections and by performing element balance computations across the major species (Oßwald et al. (2014); Kasper et al. (2009); Schenk et al. (2013); Osswald et al. (2007)). The distinctive advantage of the PEPICO method is to unveil isomeric species using their photoelectron spectrum as identification criterion in addition to the PI spectrum (Oßwald et al. (2014); Krüger et al. (2014); Pieper et al. (2018); Bodi et al. (2013)). Unambiguous identification is an important aspect for quantification, since identification is an imperative prerequisite for accurate subtraction procedures for mass-convoluted species. Advantages of PEPICO for quantitative combustion diagnostics have been thoroughly demonstrated by Felsman et al. and Oßwald et al. (Felsmann et al. (2016); Oßwald et al. (2014)). Quantification of isomers based on species selection using the photoelectron signal has also been demonstrated by Oßwald et al. and can be used to a limited extend (Oßwald et al. (2014)).

A PEPICO flame experiment has been operated at the VUV beamline of the Swiss Light Source (SLS) for several years (Krüger et al. (2018); Bierkandt et al. (2017); Felsmann et al. (2015); Oßwald et al. (2014); Krüger et al. (2019)). The experiment is coupled to an imaging/double imaging photoelectron photoion coincidence spectrometer (iPEPICO/i²PEPICO) (Bodi et al. (2009); Bodi et al. (2012); Oßwald et al. (2014); Sztáray et al. (2017)). Flame experiments have been performed at the Advanced Light 
Source (ALS), Berkeley, USA (Cool et al., 2005; Hansen et al., 2009) and the National Synchrotron Laboratory (NSRL), Hefei, China (Qi (2013)) using PI-MBMS, while another double imaging PEPICO combustion experiment is available at the SOLEIL synchrotron, Saint-Aubin, France (Garcia et al. (2013); Krüger et al. (2014)).

Another class of combustion experiments uses atmospheric and low-pressure flow reactors ( $\mathrm{Q} i$ (2013); Moshammer et al. (2015); Moshammer et al. (2016)) coupled to PI-MBMS. These experiments allow the investigation of low-temperature combustion chemistry in oxidizing and nonoxidizing environments, while capitalizing on the distinct advantages of PI-MBMS. The well-stirred reactor first described by Matras and Villermaux (Matras \& Villermaux (1973)) has been extensively used in these experiments. It uses gas jets to mix the reactants continuously inside a heated glass sphere, where reactions take place. Currently, such experiments are performed at the ALS (Moshammer et al. (2015); Moshammer et al. (2016)) and at the NSRL (Herbinet et al. (2011)).

Although combustion experiments coupled to PI-MBMS have been used to investigate a wide range of combustion conditions and species, they have been limited to pressures up to one atmosphere until now (Moshammer et al. (2016); Moshammer et al. (2015); Hansen et al. (2009); Cool, Mcllroy, et al. (2005); Qi (2013); Krüger et al. (2014); Sztáray et al. (2017); Oßwald et al. (2014)). To get comprehensive speciation data for low-temperature combustion experiments across a wider pressure range, a high-pressure plug-flow reactor experiment has been commissioned at the VUV beamline of SLS using the CRF-PEPICO (Combustion Reactions Followed by Photoelectron Photoion Coincidence) spectrometer (Sztáray et al. (2017)). Similar pressurized plug-flow reactors have been used to generate quantitative speciation data for reaction mechanism development at elevated pressures using different analytics such as gas chromatography and molecular-beam sampling (e.g. (Rasmussen et al. (2008); Kaczmarek et al. (2019b); Kaczmarek et al. (2019a))).

This work describes the new experimental setup, the interface to the endstation, and first results. The aim of the experiment is to use the ability of the $i^{2} P E P I C O$ method to distinguish convoluted species, especially isomers and to provide comprehensive isomeric species identification using photoionization spectra and ms-TPES. In addition, quantification of combustion species is demonstrated, which provides an indispensable capability for reaction mechanism development and validation. In a first campaign, measurements of different diluted reactant mixtures were performed with the new pressurized reactor setup. Some data from an $n$-heptane doped methane condition (cf. Table 1) will be used to demonstrate the capability of the new experiment.

\section{EXPERIMENTAL SETUP}

\section{A. Pressurized flow reactor}

A pressurized plug-flow reactor interfaced to the CRF-PEPICO spectrometer is used to investigate the conversion reactions of fuel and oxidizer at a pressure of 6 bar. Plug-flow operation of the reactor is ensured by using an appropriate flow regime (280 sccm total flow in this case). This ensures the applicability of one-dimensional combustion simulations. The flow reactor is integrated into an expansion chamber, leading to the molecular-beam interface. The first expansion stage consists of a manual needle valve (cf. Figure 2) (GB1H-6M, Hy-Lok, Songjeong-Dong, South Korea). A cutaway view of the expansion chamber setup and the reactor integration is shown in Figure 1. The reactor is connected to the expansion stage via heated stainless steel pipes of $12 \mathrm{~mm}$ outer diameter (OD) and $6 \mathrm{~mm}$ OD for the needle valve stage. Both pipes have a wall thickness of $1 \mathrm{~mm}$, providing flow volumes of inner diameter (ID) of 10 and $4 \mathrm{~mm}$, respectively. Temperatures of the connecting pipes are kept at $373-423 \mathrm{~K}$ to prevent condensation of water and other products, while keeping the temperature low enough to prevent further reactions of species in the stream discharged from the 
reactor. Heating is provided by electrical heating tapes (Type HSQ, HORST GmbH, Lorsch, Germany) controlled by a digital PID heating controller.

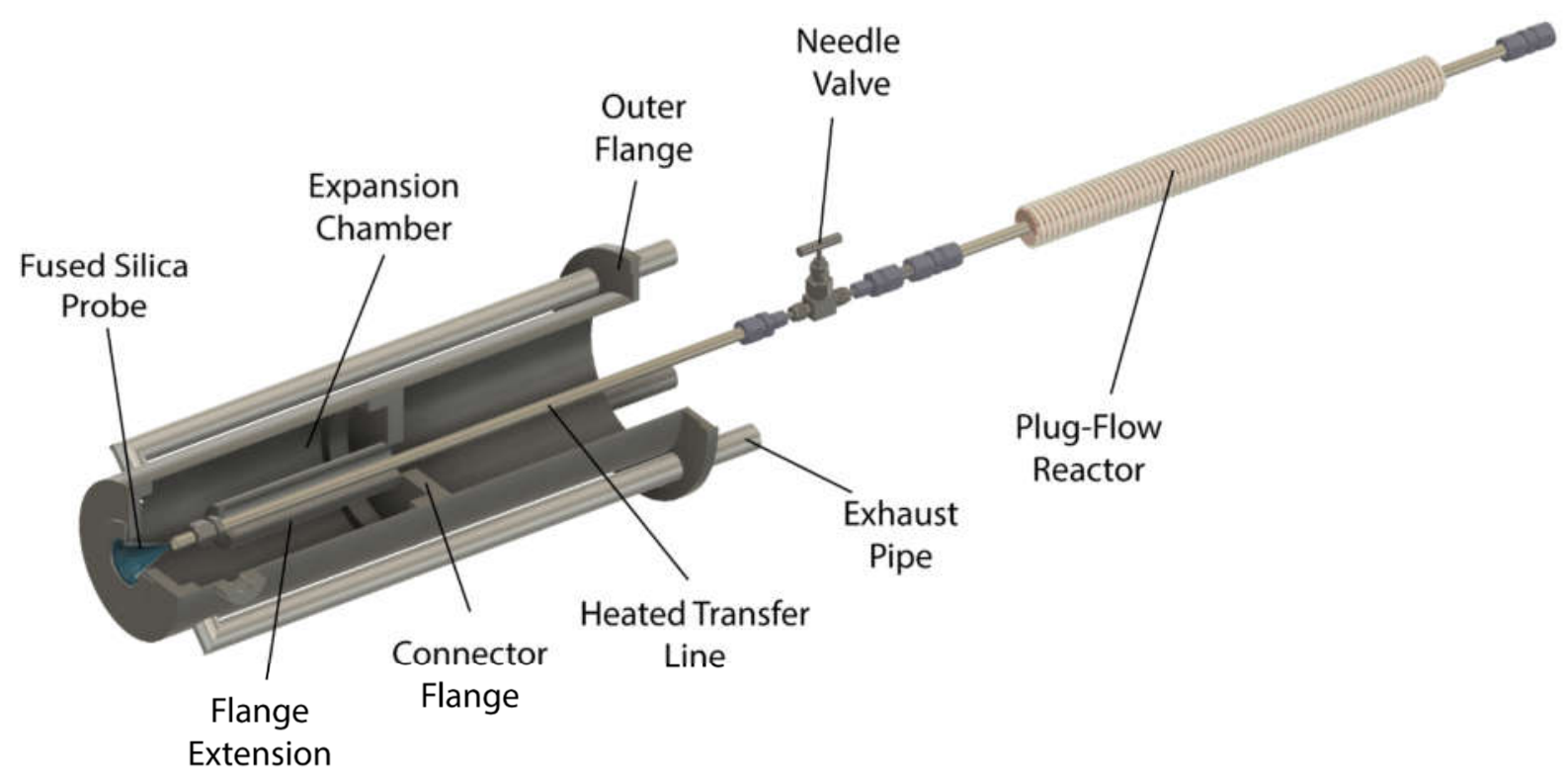

Figure 1: Cutaway view of the expansion chamber integration of the reactor experiment and first stage of the sampling interface. Transfer line heating and insulation of the transfer line and the reactor are omitted for clarity.

Gases expand through the transfer line into the expansion chamber, which contains the fused silica sampling probe. A long transfer line is generally undesirable, because wall interactions and secondary, catalytic reactions may affect the sample composition. However, the molecular-beam skimmer is located approx. $500 \mathrm{~mm}$ from the outer flange of the expansion chamber (cf. (Bodi et al. (2012); Oßwald et al. (2014))), which required the use of a longer than ideal heated transfer line. It guides the gases directly to the orifice of the fused silica probe. Heating as close as possible to the sampling point is provided by a flange extension of the connector flange (cf. Figure 1) reaching into the expansion chamber. Heating tape is wrapped around the $12 \mathrm{~mm}$ OD stainless steel transfer pipe on the atmospheric side of the flange and around the needle valve all the way to the copper mantle. The transfer pipe and heating tape are insulated using multiple layers of fiberglass insulation wrapping. Insulation and transfer line heating are omitted in Figure 1 for clarity.

A detailed cutaway view of the reactor internals and the throttling stage is shown in Figure 2 . The reactor consists of an outer pressure shell made of a $12 \mathrm{~mm}$ OD AISI 316 stainless steel pipe with a wall thickness of $1 \mathrm{~mm}$ and a length of $650 \mathrm{~mm}$ that serves as pressure containment. To prevent wall interactions, the heated flow volume contains an inner liner made of a fused silica pipe with an OD of $8 \mathrm{~mm}$ and a wall thickness of $1 \mathrm{~mm}$. The liner provides chemically inert, hydraulically smooth walls that cover the outer shell length of $650 \mathrm{~mm}$ completely. The fused silica liner is loosely sealed against the stainless steel outer pressure containment on both ends of the reactor using polytetrafluoroethylene (PTFE) tape. This prevents the gases from bypassing the fused silica liner and reacting in the gap between liner and outer shell, while allowing sufficient leakage rate to equalize gap pressure. 


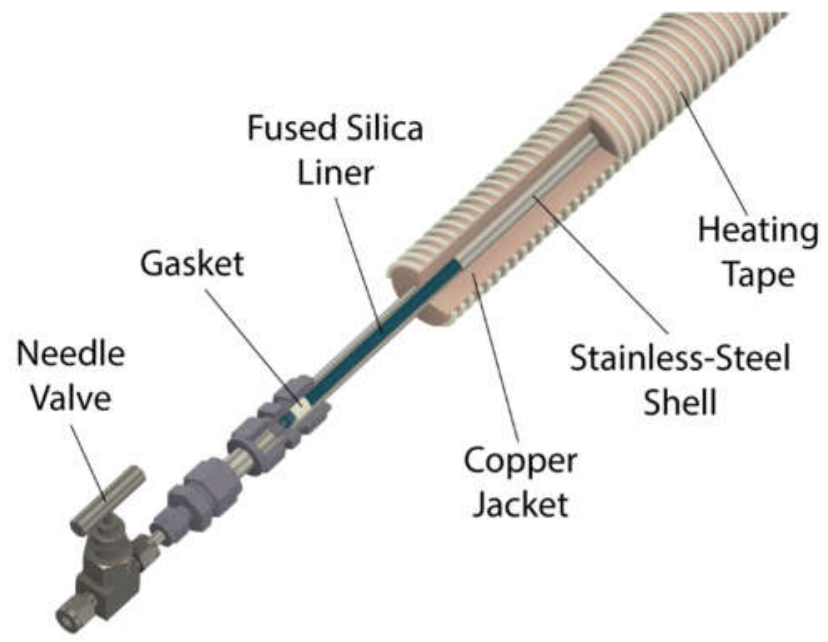

Figure 2: Cutaway detail of the reactor stage internals and expansion stage showing the PTFE seal between liner and outer shell, the arrangement of the electrically heated copper jacket around the isothermal region of the reactor, and the needle valve expansion stage. Insulation of the reactor is omitted for clarity (details in text).

The outer stainless steel shell is enclosed by a copper jacket of OD of $35 \mathrm{~mm}$ and a length of $450 \mathrm{~mm}$, which distributes the electrical heating power evenly. The copper mantle is split along a horizontal line for assembly around the stainless steel outer reactor shell. Measured temperature profiles of the reactor design for set temperatures of 473-973 K show a nearly perfect isothermal zone (Kaczmarek et al. (2019a); Kaczmarek et al. (2019b)). The isothermal zone stretches the full $450 \mathrm{~mm}$ length of the copper mantle, while temperature ramps are present in the lead-in and lead-out zones both stretching the remaining $100 \mathrm{~mm}$ on each end of the reactor. The reactor is operated in constant flow regime, which leads to lower residence times with increasing temperature.

The round pocket in the copper mantle, in which the stainless steel pipe is embedded, is manufactured with a tight fit to the $12 \mathrm{~mm}$ pipe. The surface contact between heating mantle and the outer stainless steel reactor shell provides satisfactory thermal conductivity between the components. The copper mantle is wrapped with two electrical heating tapes (Type HSQ, HORST $\mathrm{GmbH}$, Lorsch, Germany) of $6000 \mathrm{~mm}$ length each, side-by-side. Each tape is connected to a digital PID temperature controller (HT MC11, HORST GmbH, Lorsch, Germany) and the temperature is measured using two mineral insulated type $\mathrm{K}$ thermocouples (TC Mess- und Regeltechnik GmbH, Mönchengladbach, Germany) inserted into $3 \mathrm{~mm}$ drilled holes in the heat distribution jacket at 25\% and $75 \%$ length position. The reactor is wrapped in several layers of fiberglass tape insulation and a layer of refractory ceramic fiber insulation. To contain the fibrous insulation material, facilitate handling of the reactor, and to provide a final convective barrier, the outer insulation layer consists of laboratory aluminum foil. All insulation layers are omitted in Figure 1 andFigure 2 for clarity. Fluid connections are made using compression fittings.

\section{B. Fluid supply systems and operation}

A detailed view of the fluid supply of the setup can be seen in Figure 3. Only the fluid systems are shown, electrical wiring for the controllers and instrumentation is mostly omitted for the sake of clarity. We describe the system from the left of Figure 3, progressing to downstream components of the setup to the right. The feed system is set up for experiments using gaseous and liquid fuels with argon (or other) diluent and an oxidizer. Starting from the left of Figure 3, the supplies of gaseous fuel (F), oxidizer (O), a gaseous dopant (D), and inert diluent (AR) are shown. The liquid feed system 
for liquid fuels and dopants is shown on the bottom left of Figure 3, with liquid feedstock coming from a reservoir (L).

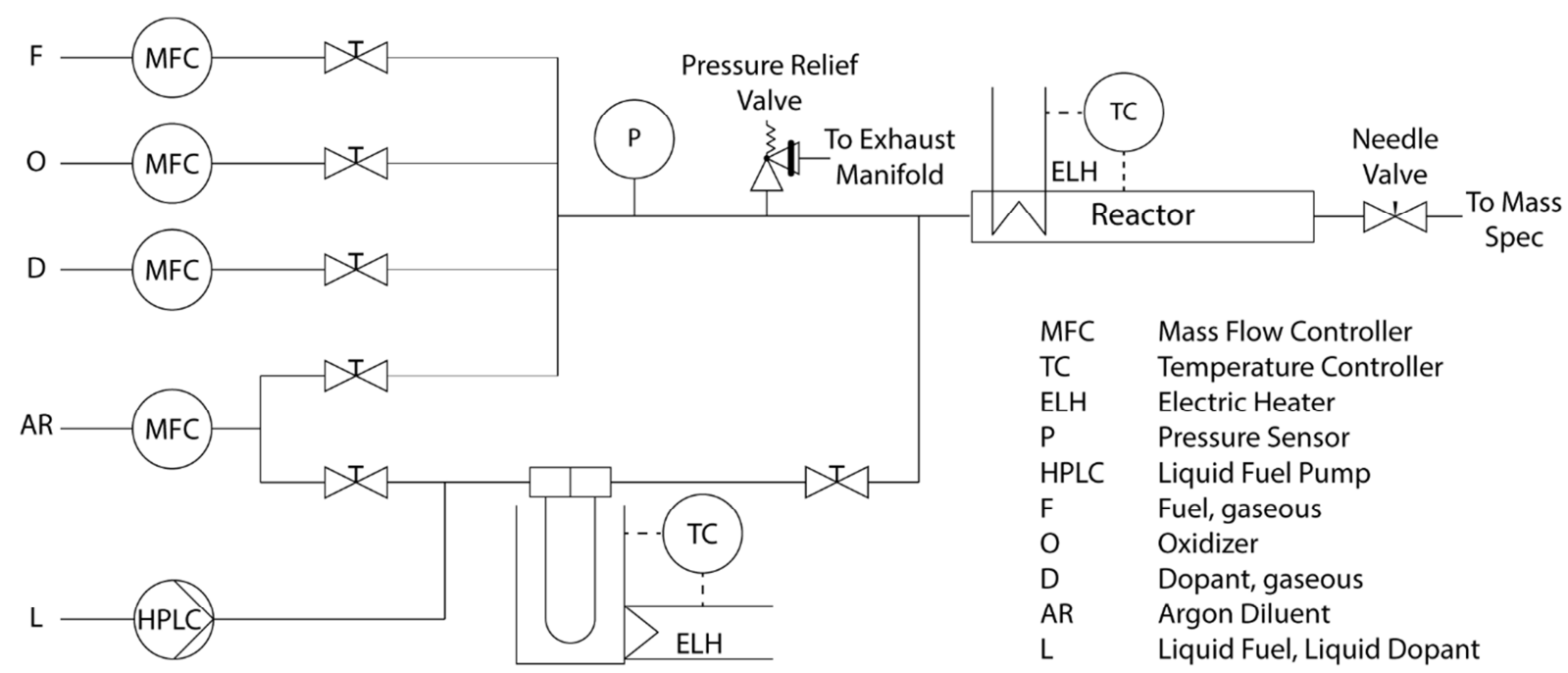

Figure 3: Diagram of fluid supply and metering system employed to condition the reactant flow to the plug-flow reactor stage. Major components are displayed with symbols partially based on ISO 10628-2.

The gaseous reactants are fed from gas cylinders. Pressure is reduced by commercial pressure regulators to a foreline pressure of approximately 7 bar total. Thermal mass flow controllers (MFCs) (MKS Instruments Inc., Andover, MA, USA) meter gases. All gaseous components are fed through manual isolation valves that allow for isolation of the individual feed streams from the downstream components and the reactor. Gaseous components are transferred to a mixing point where they intermix by diffusion. The liquid feed system uses the same MFC to supply the argon diluent as in experiments with solely gaseous fuels. When operating the liquid evaporation system, the alternate argon supply line is isolated from the setup and the entire argon dilution gas stream is delivered to the evaporator.

A high-pressure liquid chromatography pump (HPLC) (Bischoff Analysetechnik u. -geräte GmbH, Leonberg, Germany) feeds and meters liquid fuels and dopants to the evaporator consisting of a multiple-bend stainless steel U-pipe. Condensation of the liquid fuel in downstream lines is suppressed by keeping the partial pressure of evaporated liquid below its vapor pressure. With this method, the gas stream is not saturated for the given temperature and the introduced fuel is directly metered by the HPLC pump instead. If a higher concentration is desired, the unheated line can be replaced by a heated foreline to the reactor, which increases the fuel vapor carrying capacity of the dilution gas stream. The evaporator is kept at constant temperature, above the evaporation temperature of the liquid fuel in an electrically heated and temperature-controlled bath of silicone oil (thermostat; Julabo GmbH, Seelbach, Germany). The HPLC is used to deliver liquid reactant with sufficient pressure for introduction into the pressurized diluent stream and both streams are fed to the evaporator column. The mixed gas stream is then delivered to the mixing point in front of the reactor and intermixed with the remaining gaseous component stream. There are isolation valves in front of and behind the evaporator column. For experiments using gaseous fuels only, the evaporator is isolated from the reactor feed line and argon supply. The feed line connecting the flow controller rig with the reactor is equipped with an adjustable relief valve (Hy-Lok, Songjeong-Dong, South Korea) that opens in case of overpressure in the feed line due to incorrect pressure settings or 
ignition events in the reaction zone. In normal operation, the safety valve is set to a pressure slightly above the desired pressure for the experiment. The normally closed valve releases any overpressure into the exhaust manifold in case of a blow-off event. The reactor setup with its electrical heating and heating control is also shown as part of the fluid system up to the spectrometer. Behind the reactor, a manual needle valve acts as a throttling stage, followed by the low-pressure stages leading to the $i^{2} P E P I C O$ spectrometer. This constitutes a limitation of the setup, because transient species can be destroyed during expansion in the valve stage.

\section{CRF-PEPICO endstation, $\mathrm{x} 04 \mathrm{db}$ (VUV) beamline, and experiment integration}

The analytical stage used for the experiment is the double imaging CRF-PEPICO endstation as described by Sztáray et al. (Sztáray et al. (2017)). A brief description of the system, its capabilities and the accompanying beamline that delivers the VUV light for ionization is given here. For additional information the reader is referred to the references describing these systems in more detail (Bodi et al. (2009); Bodi et al. (2012); Johnson et al. (2009); Sztáray et al. (2017)).

The major systems comprising the analytical capability provided at the SLS are the beamline (Bodi et al. (2009); Bodi et al. (2012); Johnson et al. (2009)) and the CRF-PEPICO endstation (Bodi et al. (2012); Sztáray et al. (2017)), into which the pressurized flow reactor combustion experiment is integrated. The grazing incidence monochromator is a turntable design featuring three positions to accommodate different laminar and blazed gratings. The dispersion of the laminar gratings corresponds to an energy dependent energy resolution of $E / \Delta E \approx 10^{4}$ (Bodi et al. (2009)), while the high-flux blazed grating delivers $E / \Delta E \approx 10^{3}$ (Bodi et al. (2012)) at photon energy of $10 \mathrm{eV}$. The light transfer line of the beamline is evacuated to ultrahigh vacuum to prevent loss of photon flux to absorption in the background gas. The differentially pumped gas filter houses the exit slit and is situated directly in front of the ionization chamber, at a distance of $500 \mathrm{~mm}$ from the ionization region (Bodi et al. (2012)). It eliminates higher VUV harmonics passed by the monochromator by absorption in a rare gas mixture (Bodi et al. (2009)). In the measurements reported here, for energies $h v>15 \mathrm{eV}$, the gas filter is filled with neon, while at energies of 10.6-15.0 eV, the filter utilizes a $75 \%$ neon, $25 \%$ argon mix (Bodi et al. (2009)). For $h v<10.6 \mathrm{eV}$, higher harmonics are filtered by a $\mathrm{MgF}_{2}$ window, which can be manually inserted into the beam line using a screw operated shutter stage. The beamline delivers an energy dependent photon flux to the ionization region with a maximum spot size of $4 \times 2 \mathrm{~mm}^{2}$ (Bodi et al. (2009); Bodi et al. (2012)).

The CRF-PEPICO endstation affords simultaneous analysis of photoelectrons and time-of-flight (ToF) analysis of positive ions at a high count rate of up to $10^{5} \mathrm{~s}^{-1}$ (Bodi et al. (2009)). The instrument uses two stage linear ion extraction which allows electron imaging and $1^{\text {st }}$ order space focusing ion detection, adhering to Wiley-McLaren conditions (Bodi et al. (2012); Wiley \& McLaren (1955)) and double imaging velocity map imaging operation (Sztáray et al. (2017); Bodi et al. (2012)). Ions and electrons are continuously extracted from the interaction region towards two RoentDek DLD 40 imaging detectors (RoentDek Handels $\mathrm{GmbH}$, Kelkheim, Germany) utilizing delay lines that allow fast position sensitive detection of photoelectrons and photoions (Bodi et al. (2012)). Every coinciding electron and ion event is individually matched. The detected electron events are utilized to start ToF measurement of positive ions. Data acquisition takes place with a PSI in-house developed software.

In the system overview in Figure 4, the mass spectrometer (MS) is shown schematically to the left, with the reactor integration into the source and expansion chambers shown to the right. The interaction of expansion stages with pressures ranging from 6 bar upstream of the needle valve down to experimental chamber pressures in the beam interaction region is also visible in Figure 4. The molecular beam (red) is formed by the fused silica probe and collimated by a nickel molecularbeam skimmer with an orifice diameter of $2 \mathrm{~mm}$ (Beam Dynamics Inc., Jacksonville, Florida, USA). 
The skimmer orifice transfers the sample into the experimental chamber where the orthogonal VUV light beam ionizes the sample. Velocity map imaging photoelectron and photoion detection is shown schematically in Figure 4 and representative false color images of intensity distributions on the imaging detectors are shown.

During the reactor campaign, the experimental chamber was pumped by a cryogenic Leybold COOLVAC $1500 \mathrm{CL}$. The source chamber was held at the desired pressure in the region of $3 \times 10^{-5}$ mbar by a COOLVAC 5000 CL cryogenic pump and two turbo pumps (Pfeiffer TPH 1201 UP and HiPace 1200).

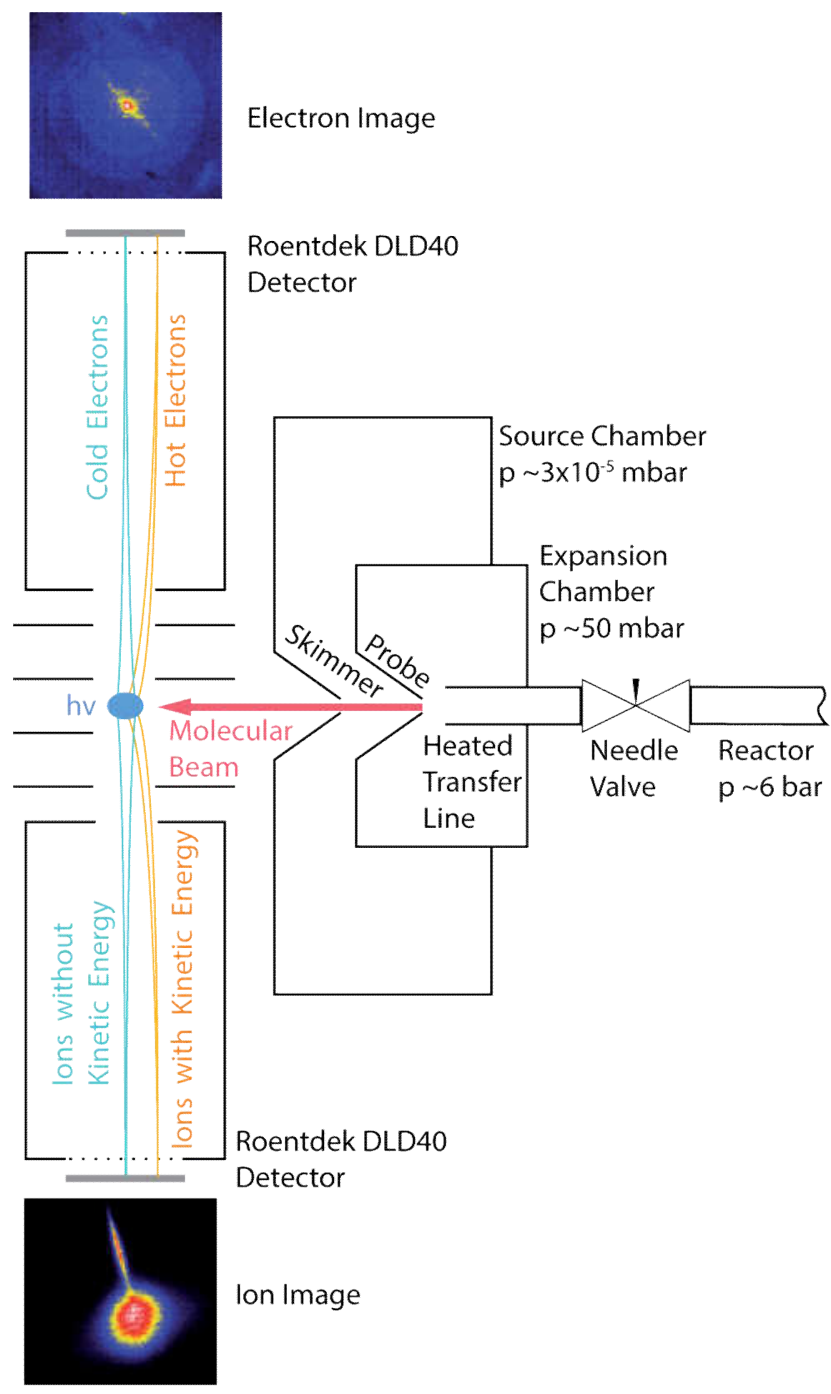

Figure 4: Schematic view of CRF-PEPICO spectrometer in double imaging operation (Bodi et al. (2012); Sztáray et al. (2017)), experiment integration and differential pumping stages used to bring the pressure from reactor pressure ( 6 bar) down to ionization chamber pressure $\left(\approx 3 \times 10^{-6} \mathrm{mbar}\right)$ in three expansion stages. The light beam enters the ionization region normal to plane of illustration, denoted $h v$, in blue. Representative false color intensity maps showing photoelectron and photoion velocity map images are provided at the top and bottom of the schematic. 
After the reactant stream, at a pressure of 6 bar, passes the needle valve that comprises the first expansion stage, it enters the expansion chamber (cf. Figure 1, Figure 2). This chamber is also used for the original flame experiment (Oßwald et al. (2014)) and is pumped by a Pfeiffer Adixen ACP 120 G Roots pump. The expansion chamber is kept at a constant operating pressure of $50 \mathrm{mbar}$ by an electrically driven butterfly throttle valve controlled by a digital/analog pressure controller (MKS Instruments, Andover, MA, USA). The controller reads the chamber pressure by means of a Baratron rough vacuum gage (MKS Instruments, Andover, MA, USA). The fused silica molecular-beam sampling probe has an orifice diameter of $320 \mu \mathrm{m}$. The pressure in the expansion chamber is chosen to provide an acceptable source chamber pressure of ca. $3 \times 10^{-5} \mathrm{mbar}$. The molecular beam enters the ionization chamber and its scattering contributes to the background pressure of approx. $3 \times 10^{-6}$ mbar during experiments.

\section{EXPERIMENTAL}

\section{A. Overview and motivation of experiments}

The reactor setup can accommodate experiments at pressures ranging from 0 to 6 bar total at temperatures between room temperature and $973 \mathrm{~K}$. Such conditions allow the investigation of partial oxidation and fuel conversion in the low-temperature range at pressures relevant for technical combustion processes. Fuel supply systems accommodate the investigation of multi-fuel reactant mixes containing liquid and gaseous fuels as well as dopants. In the first campaign reported here, we have investigated several combustion process conditions using the novel pressurized reactor setup interfaced with the CRF-PEPICO endstation. First results from a conversion experiment using methane $\left(\mathrm{CH}_{4}\right)$ doped with n-heptane $\left(\mathrm{C}_{7} \mathrm{H}_{16}\right)$ as fuels at an equivalence ratio of 8 and diluted with $82.8 \%$ argon are presented here, because they are best suited to demonstrate the isomer resolution capabilities of the experiment. Mole fractions and reactant flows for the condition are given in Table 1. The argon dilution is relatively low due to a minimum flow limitation of the liquid fuel delivery pump. The small amount of dopant would not have been possible at higher dilutions. The addition of highly reactive $n$-heptane reduces the temperatures required for the onset of reactions converting the stable hydrocarbon $\mathrm{CH}_{4}$. Similar conditions have been investigated with the goal of providing validation data for reaction mechanism development for hydrocarbon conversion (Kaczmarek et al. (2019a)).

\begin{tabular}{ccc} 
Reactant & Flow $[\mathrm{sccm}]$ & Mole Fraction $\mathrm{x}$ \\
\hline $\mathrm{Ar}$ & 231.9 & 0.833 \\
$\mathrm{O}_{2}$ & 11.2 & 0.04 \\
$\mathrm{CH}_{4}$ & 35.1 & 0.125 \\
$\mathrm{C}_{7} \mathrm{H}_{16}$ & 1.76 & 0.006
\end{tabular}

Table 1: Compound molar fractions and flows for the $\varphi=8$ conditon of $\mathrm{CH}_{4}$ doped with $\mathrm{n}$-heptane

The key capability of VUV photoionization to detect species selectively in complex reactive environments is demonstrated by means of ms-TPES and photoionization spectra. Isomer-selective species identification is shown for two isomers at $m / z$ 72. The main species, namely the reactants $\mathrm{O}_{2}$, $\mathrm{CH}_{4}$, and $\mathrm{n}$-heptane as well as the major products $\mathrm{CO}, \mathrm{CO}_{2}$, and water are quantified at the conditions in Table 1. Quantification of the low-temperature intermediates methanol $\left(\mathrm{CH}_{3} \mathrm{OH}\right)$ and acetaldehyde $\left(\mathrm{CH}_{3} \mathrm{CHO}\right)$ is performed. Mole fraction profiles are compared to a recent combustion mechanism (Zhang et al. (2016)) and GC reference data (Kaczmarek et al. (2019a)). The comparison serves to demonstrate the quality of the quantitative data for reaction mechanism development. 


\section{B. Measurement procedure and reactor operation}

The pressure in the isothermal reaction zone is kept constant by pre-setting it using the manual needle valve. Temperatures are scanned starting from the lowest desired temperature (in case of the discussed condition $473 \mathrm{~K}$ ). The temperature is increased subsequently in steps of $50 \mathrm{~K}$ up to a maximum temperature of $973 \mathrm{~K}$. For each step, mass spectra are obtained at different energies in the range of compound ionization energies (IEs) of the species of interest. After the initial temperature ramp (temperature scans), spectra (photon energy scans) are obtained at constant temperature. Selected temperatures for the energy scans are those, which show maximum yield of species of interest during the temperature ramp measurements. These temperatures usually are in the vicinity of the reaction onset temperature of the investigated condition.

\section{Separation of isomeric species using threshold photoelectron and photoionization spectra}

The main multiplexing advantage of PI-MBMS in general and more specifically synchrotron photoionization is the ability to distinguish species by their mass and their IE (Cool, Mcllroy, et al. (2005)). The IE affords an indispensable secondary selection criterion for the discrimination of compounds. Especially for the analysis of complex species compositions, which are commonly encountered in combustion experiments, photoionization is a powerful tool (Cool, Mcllroy, et al. (2005); Hansen et al. (2009); Qi (2013)). In conventional PI-MBMS time-of-flight (ToF) mass spectrometry, species identification is performed by photoionization spectra. Consecutive spectra are taken at a single height above burner in the flame or a single temperature for reactor experiments, while the photon energy is scanned in small intervals. For a single species, no signal will be acquired while the set energy of the ionizing VUV light is below the IE of the molecule. When the ionization threshold is reached a signal is acquired whose intensity increases with increasing photon energy. The signal intensity increases until the dissociative photoionization of the molecule surpasses the increase in ionization cross section as a function of the photon energy. Species identification is performed by comparing the IE at the onset of the steepest slope of the PI spectrum to literature or calculated IE values. For convoluted species such as isomers, the additional signal contribution of the isomer with the higher IE creates a further increase in the slope of the ion yield curve. Isomers can be selectively detected by their differing ionization energies. The differing contributions to the PI spectrum can be used to deduce information about ratios of contributing species. These principles were first demonstrated for combustion experiments by Cool et al. in 2005 (Cool, Mcllroy, et al. (2005)).

Using double imaging PEPICO mass spectrometry, isomer and species identification can in general be performed in the same manner. Moreover, the distinct resonance pattern given by the simultaneously acquired photoelectron spectra result in a unique fingerprint of the species (Oßwald et al. (2014); Bodi et al. (2013)). By comparing the measured threshold photoelectron spectrum with literature reference photoelectron spectra and Franck-Condon simulations, a confident identification of species at the same integer mass, such as isomers, can be performed (Oßwald et al. (2014); Krüger et al. (2014); Pieper et al. (2018); Bodi et al. (2013)). The same principle applies for mass-convoluted non-isomeric species, which can occur frequently due to the low mass resolution of the instrument. The use of imaging PEPICO for acquisition of flame data was first demonstrated by Oßwald et al. (Oßwald et al. (2014)) at the iPEPICO endstation at SLS and later by Krüger et al. (Krüger et al. (2014)) and Pieper et al. (Pieper et al. (2018)) at the DELICIOUS III double imaging PEPICO setup at Soleil (Garcia et al. (2013)). Distinct advantages of using double imaging for flame diagnostics and analysis methods specific to double imaging PEPICO have been demonstrated (Krüger et al. (2019); Felsmann et al. (2016)) since. Krüger et al. demonstrated that the ion image can be used to distinguish species in the thermalized background and in the molecular beam, specifically for the case of the transient 
$\mathrm{OH}$ radical in combustion experiments (Krüger et al. (2019)). A summary of double imaging valence photoionization coincidence techniques and accompanying analysis methods can also be found in Baer and Tuckett (Baer \& Tuckett (2017)).

For species identification and fingerprinting using PI spectrum and ms-TPES, the peak at $m / z 72$ is selected. The signal is likely to arise from a convolution of species directly from the major fuel destruction pathways of $n$-heptane (Ranzi et al. (1995); Curran et al. (1998); Pelucchi et al. (2014); Luck et al. (1973)). The energy scan was performed between photon energies of $9.0 \mathrm{eV}$ and $10.3 \mathrm{eV}$ with intervals $\Delta E=0.025 \mathrm{eV}$ (cf. Figure 5). Potential species at lower ionization energies are tetrahydrofuran with an IE close to $9.40 \mathrm{eV}$ (Behan et al. (1976); Doucet et al. (1972); Hernandez (1963)) and the likely species 2-butanone (IE $=9.52 \mathrm{eV}($ Traeger (1984))), iso-butanal (IE $=9.71 \mathrm{eV}$ (Hernandez et al. (1977))), and n-butanal (IE = $9.83 \mathrm{eV}$ (Traeger \& McAdoo (1986))). At higher photon energies, dimethyloxirane isomers may be detected, although they are unlikely to be contributing to the ms-TPES, since their IE is close to the maximum energy of the energy scan (10.3 eV) near $10.0 \mathrm{eV}$ (Eskola et al. (2017)). Moreover, dimethyloxiranes are unstable and hence are likely destroyed in the expansion stage. n-Pentane and 2-methylbutane can both be excluded as contributors to the photoionization spectrum due to their IEs being close to or above the maximum photon energy of the scan (Watanabe et al. (1962)).

Ketones and aldehydes are likely species encountered in the sample arising from n-heptane destruction, since they are produced by major low-temperature fuel oxidation and destruction pathways as described in modeling studies by multiple authors (Pelucchi et al. (2014); Curran et al. (1998); Ranzi et al. (1995)). Lumped schemes for the initiation of $n$-heptane destruction at low temperatures lead through formation of peroxides and subsequently ketohydroperoxides (Ranzi et al. (1995); Pelucchi et al. (2014); Curran et al. (1998)), which then branch into lower hydrocarbon ketones and aldehydes (Ranzi et al. (1995); Curran et al. (1998); Luck et al. (1973)).

Panel (a) of Figure 5 shows the PI spectrum and marked literature reference ionization energies (Hernandez (1963); Doucet et al. (1972); Behan et al. (1976); Traeger (1984); Hernandez et al. (1977); Traeger \& McAdoo (1986)). The late onset of the PI spectrum shows no evidence for the presence of tetrahydrofuran. However, the increase in signal and thus ion yield matches the IE of 2-butanone. The reason for the signal increase preceding the marked adiabatic IE can be seen in the photoelectron spectrum (Figure 5b), where the photoelectron yield increases prior to the adiabatic IE value. Moreover, another contributing factor is that the adiabatic value is particularly close to the vertical IE for this species (Lindstrom et al. (1997)). A distinctive feature is visible for $n$-butanal, while iso-butanal shows no feature in the PI spectrum in Figure 5 (a). Hence, 2-butanone and n-butanal are likely constituents of the sample injected into the spectrometer.

Further and much clearer confirmation of this assignment is given by the data presented in panel (b) of Figure 5. It shows the measured ms-TPES and reference spectra for $m / z 72$ species from Pieper et al. (Pieper et al. (2018)). Here, the data show no distinctive features of either tetrahydrofuran or isobutanal, while features consistent with 2-butanone and n-butanal are visible when compared with the reference spectra. The reference spectrum for 2-butanone (Pieper et al. (2018)) required a slight shift of approx. $20 \mathrm{meV}$, to accommodate for a peak position discrepancy. It shows a red shift with respect to the experiment, which may e.g. be caused by calibration, the Stark shift, or other contributing factors. The shape of the reference spectrum in comparison to the experiment allows identification with great certainty due to the excellent agreement in the vibrational progression. Identification of species was feasible using ms-TPES in spite of the high number of possibly convoluted species and the special challenge that arises since many of these species do not show very distinct spectra (Pieper et al. (2018)). iso-Butanal may only be produced below the detection 
limit, as no feature hinting at this intermediate is visible in Figure 5. This observation is also confirmed by GC data (cf. (Kaczmarek et al. (2019a))).

For the sake of completeness, it has to be mentioned that more isomers exist at $m / z 72$, such as the stereoisomers of crotyl alcohol and other butenols. They show no significant contribution to the signal as the vertical IE in available reference spectra of 3-buten-1-ol and 3-buten-2-ol (Pieper et al. (2018)) is in the vicinity of that of iso-butenol (cf. Figure 5).

Under similar process conditions, isomeric contributions can also be resolved by GC measurements. In contrast to the experiments presented here, the presence of the respective isomers must at least be suspected to allow GC measurements because the GC method and column must be matched to the separation problem. The identification of isomers using the PEPICO technique is universal and does not require prior knowledge of the sample composition when the photon energies can be scanned over a wide energy range. This aspect of the measurements makes the new experiment a valuable addition to the available analytical portfolio.

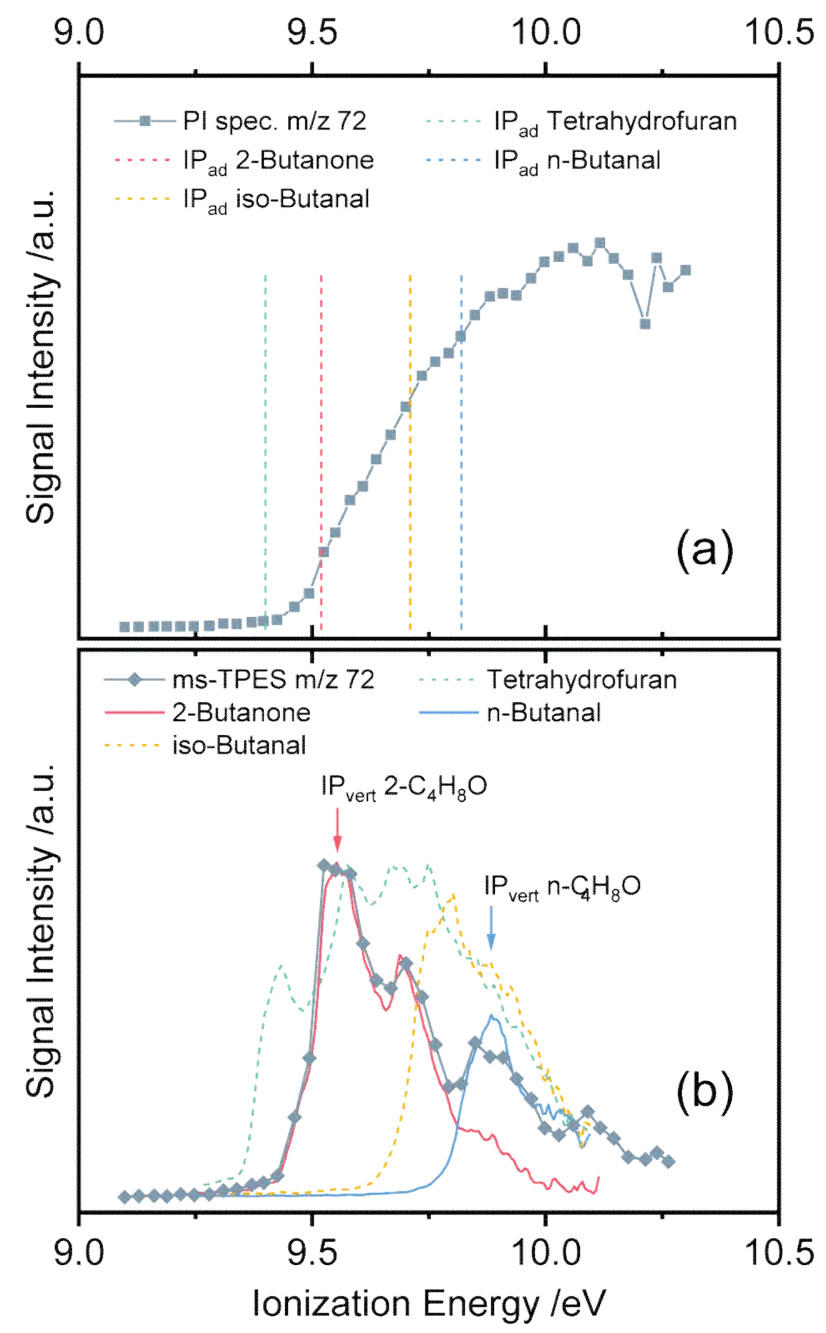

Figure 5: Photoionization spectrum (PI spec.) (a) and ms-TPES (b) of the $m / z 72$ peak and comparison to reference literature photoelectron spectra from Pieper et al. (Pieper et al. (2018)). Literature values for adiabatic ionization energies for some $m / z 72$ species candidates are displayed by dashed lines in (a). Identical species color coding is used for the TPE in panel (b). In (b), the experimental values are displayed as solid lines with symbols and compared to species present in the sample (solid lines) and undetected species (dashed lines). 


\section{Quantification of species and comparison to model simulations and reference data}

To enable comparison between experiment and chemical kinetics simulations, quantification of species is an essential capability of any analytical technique for combustion science. This encompasses requirements for reproducibility of the experimental conditions and also serves as a quality benchmark for the experiment.

The general procedure for quantification of photoionization mass spectrometry combustion data was described in detail in previous publications (Osswald et al. (2007); Kasper et al. (2009); Schenk et al. (2013); Qi (2013); Oßwald et al. (2014)). The $i^{2}$ PEPICO spectrometers peculiarities require slight modifications to the procedures which were outlined by Oßwald et al. in the work describing the flame setup originally used at SLS (Oßwald et al. (2014)).Calibration is performed according to the same principles as for photoionization flame experiments (Oßwald \& Köhler (2015)). The employment of some calibration or quantification procedure is always necessary for quantitative mass spectrometry. Different systematic (e.g. photon flux, chamber pressure, sample composition, temperature, machine parameters, properties and geometry etc.) and compound-based contributions (i.e. ionization cross sections) impact signal intensity and hence, no direct signalintensity based assertion of species concentration in the sample is possible.

To acquire mole fractions of major species in flames, element balances are established the burner surface and at a point in the exhaust gas far from the reaction zone which contains combustion intermediates (Schenk et al. (2013)). Hence, the assumption that only reactants and minor amounts of $\mathrm{CO}$ and $\mathrm{CO}_{2}$ products are signal contributors at the burner surface and only $\mathrm{CO}, \mathrm{CO}_{2}$, water, and hydrogen are contributors in the exhaust (Schenk et al. (2013)) holds. Additionally, oxygen, which can be present in the exhaust gas stream for lean flames and unburned fuel for rich flames, has to be considered. For the element balances, it is further assumed that the argon mole fraction does not change across the flame and an argon profile is later calculated using an internal calibration procedure (Schenk et al. (2013)).

Only deviations from the aforementioned procedures will be outlined in this work, as flame data approaches can generally be transferred to reactor measurements (Moshammer et al. (2016)). In contrast to flame data, the element balances for the reactor sample are established for a point at minimum temperature and maximum temperature of the measured temperature series. Here, the assumption holds that for temperatures below reaction start temperature of the investigated condition no reactions take place. Hence, the signal contributors are solely the reactants injected into the reactor, which simplifies the element balance compared to flame data evaluation. At high temperatures (maximum temperature of $973 \mathrm{~K}$ ), the assumption is made that mostly $\mathrm{CO}, \mathrm{CO}_{2}$, hydrogen, water and fuel for rich conditions, and oxygen for lean conditions, respectively, are present in the sample. This simplification introduces only minor errors in the analysis of major species mole fractions due to the low relative yield of other species at high temperatures. A further simplification of the general procedure arises, since the measured reactor conditions are highly diluted with inert gas. Under these conditions, it can be assumed that the change in argon mole fraction is negligible and the calculation of an argon mole fraction profile can be omitted. The argon mole fraction is fixed at inlet conditions for calculation of species mole fractions. In some cases however, determining an argon profile from internal calibration may be useful (Oßwald \& Köhler (2015)).

To demonstrate the ability of the experiment to generate quantitative speciation data of elevated pressure combustion, the major species $\mathrm{CO}, \mathrm{CO}_{2}$, water, oxygen and the two fuels are quantified. The data measured with the CRF-PEPICO are compared to a previously published reference measurement using gas chromatography (GC) (Kaczmarek et al. (2019a)) and to a recent combustion mechanism by 
Zhang et al. (Zhang et al. (2016)). The GC measurements do not include $\mathrm{H}_{2} \mathrm{O}$ due to the unavailability of a suitable column for this species at the time. The used mechanism contains 5336 reactions, 1268 species, and chemistry required for the simulation of n-heptane conversion (Zhang et al. (2016)). Simulations were performed using the plug flow module of ChemKin 19.2 (Ansys Inc. (2008)) and measured temperature profiles (Kaczmarek et al. (2019a)) at a pressure of 6 bar. Simulated temperatures correspond to the temperatures at which the measurements were taken.

Figure 6 shows the results of the element balancing procedure. $\mathrm{CO}, \mathrm{CO}_{2}, \mathrm{H}_{2} \mathrm{O}$, and $\mathrm{CH}_{4}$ profiles are quantified from the $h v=15.0 \mathrm{eV}$ measurement with an acquisition time of $60 \mathrm{~s}$. The $\mathrm{C}_{7} \mathrm{H}_{16}$ signal was analyzed using a temperature scan at $h v=10.3 \mathrm{eV}$ and an acquisition time of $300 \mathrm{~s}$. The argon signal was taken from a scan at $h v=15.8 \mathrm{eV}$, only slightly above the ionization threshold of argon, since the signal yield was comparatively large because of the large mole fraction of the argon diluent. Although $\mathrm{CO}_{2}$ was only present in relatively minor amounts, it was used for quantification since a direct calibration of the $\mathrm{CO}$ and $\mathrm{CO}_{2}$ signals was performed yielding more accurate results in the elemental balance computation. Hydrogen was omitted since it can directly be solved for from the other balanced species and consequently has no influence on the balance used for the profiles shown in Figure 6.
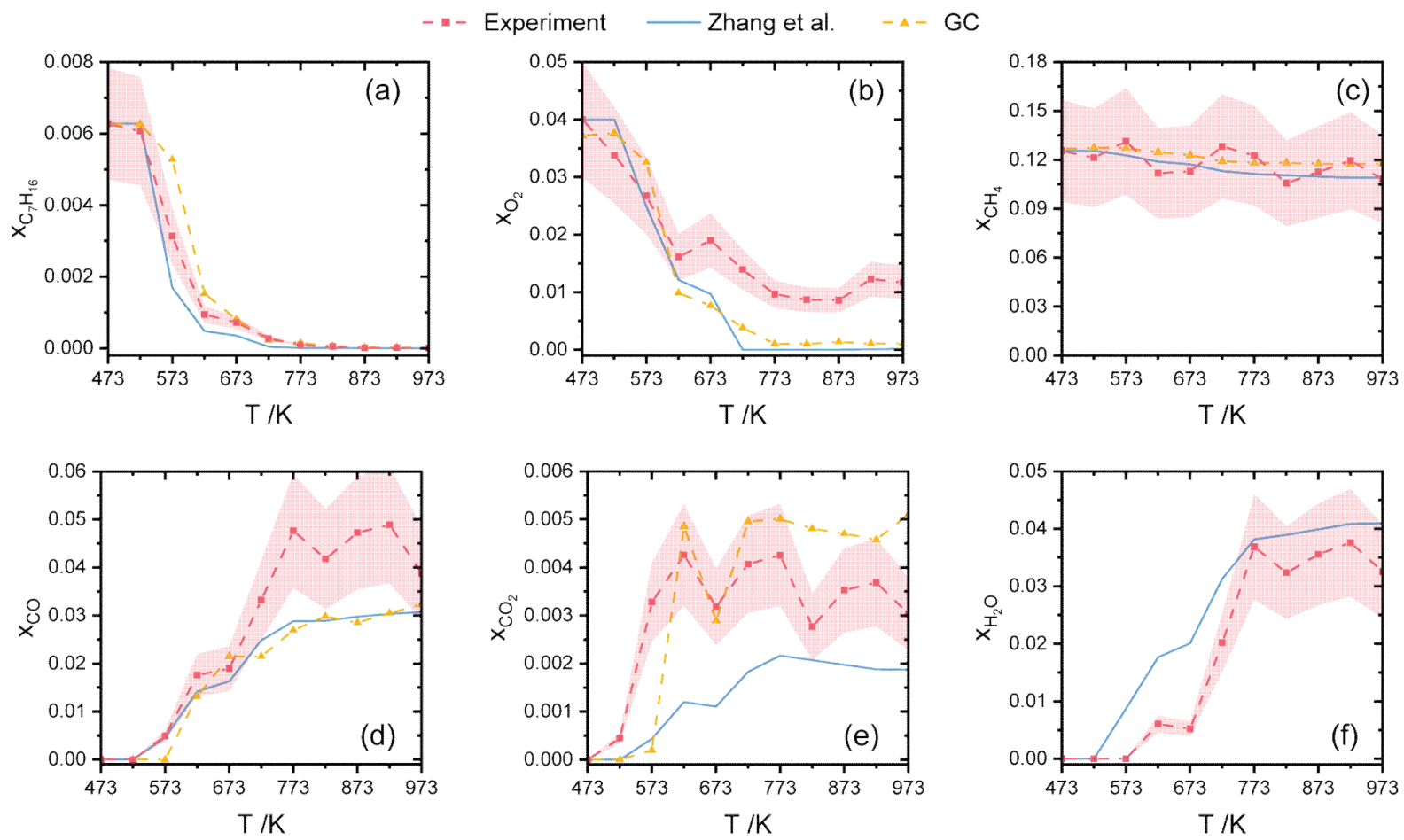

Figure 6: Mole fraction profiles of $\mathrm{C}_{7} \mathrm{H}_{16}(\mathrm{a}), \mathrm{O}_{2}(\mathrm{~b}), \mathrm{CH}_{4}(\mathrm{c}), \mathrm{CO}(\mathrm{d}), \mathrm{CO}_{2}(\mathrm{e})$ and $\mathrm{H}_{2} \mathrm{O}$ (f) from the element balance calculation of major species from the reactor experiment (dashed line with symbols; red; indicated error of $\mathbf{2 5} \%$ ) and comparison to the mechanism by Zhang et al. (Zhang et al. (2016)) (solid line; blue) and GC reference measurements (cf. (Kaczmarek et al. (2019b))) (dashed line with symbol; yellow).

It is clearly visible in Figure 6 that the CRF-PEPICO measurements are in very good agreement with both the GC data and the simulation. For the balanced major species in Figure 6 a $25 \%$ estimated error is indicated. Both fuels ((a), (c)) match perfectly the simulation and GC results. Minor discrepancies can be observed only for $\mathrm{O}_{2}$ (b) and $\mathrm{CO}$ (d) at higher temperatures, while $\mathrm{CO}_{2}$ (e) shows good agreement with the GC reference measurement. The $\mathrm{CO}$ signal was corrected for ethylene $\left(\mathrm{C}_{2} \mathrm{H}_{4}\right)$ contribution, a species still present in non-negligible concentration at the highest temperature 
of $973 \mathrm{~K}$ and the same mass-to-charge ratio as $\mathrm{CO}(\mathrm{m} / \mathrm{z} 28)$. The correction was performed by using the $\mathrm{m} / \mathrm{z} 28$ signal at $h v=11.2 \mathrm{eV}$ and subtracting the $\mathrm{C}_{2} \mathrm{H}_{4}$ photoionization cross section (Mackie et al. (2003)) corrected signal from the $\mathrm{m} / \mathrm{z} 28$ signal at $15.0 \mathrm{eV}$. The imaging photoelectron detector active area in conjunction with the ion optics and applied extraction field gradients employed in the instrument lead to a maximum acceptance for photoelectrons with a kinetic energy of $1.6 \mathrm{eV}$ (Oßwald et al. (2014); Bodi et al. (2012)). Thus, a significant loss of electrons for kinetic energies larger than $1.2 \mathrm{eV}$ is expected (Oßwald et al. (2014)). For photoelectron kinetic energies getting closer to this value, an increasing number of electrons are not detected. Since ion time-of-flight detection is triggered by photoelectron detection as start signal, the ion coincidence signal is lost if the electron is lost. To account for this loss of ion signal the $\alpha$ factor (cf. (1)), as introduced by Oßwald et al. (Oßwald et al. (2014)), was directly calibrated using $\mathrm{C}_{2} \mathrm{H}_{4}$. This calibration was performed in the range of energies used for acquisition of the $\mathrm{C}_{2} \mathrm{H}_{4}$ correction signal at $h v=11.2 \mathrm{eV}$ and the corrected CO signal acquired at $h v=15.0 \mathrm{eV}$. The described corrections might in fact still not eliminate all error associated with the signal convolution in the quantification. This effect may explain the discrepancy at higher temperatures in the $\mathrm{CO}$ profile, at which the $\mathrm{C}_{2} \mathrm{H}_{4}$ contribution increases, as the measurement matches the model and GC reference perfectly at lower temperatures (cf. Figure 6 (d)). The oxygen signal shows some contribution of methanol $\left(\mathrm{CH}_{3} \mathrm{OH}\right)$ for higher temperatures, which cannot be corrected for because of the lack of a suitable photoionization cross section of $\mathrm{CH}_{3} \mathrm{OH}$ at $15.0 \mathrm{eV}$ (Cool, Wang, et al. (2005); Zhou et al. (2009); Person \& Nicole (1971)). Mole fractions for lower temperatures match perfectly the chemical kinetics simulation result and GC measurement. An offset from the model and GC reference is visible in Figure 6 only for temperatures with a significant methanol contribution to the oxygen signal (cf. Figure 7). The introduced error has no influence on the element balance because it was assumed that no unbound oxygen is present at the final temperature of $973 \mathrm{~K}$, due to full consumption of oxygen at the extremely fuel rich condition of $\varphi=8$. The water profile (f) in Figure 6 shows good agreement with the model reference in both progression and concentration. GC measurements of water are typically difficult and no mole fraction values for water were reported in the GC work (Kaczmarek et al. (2019a)). The $i^{2}$ PEPCIO technique can measure water satisfactorily as can be seen from the good agreement between experimental and simulated profiles. The agreement emphasizes that in spite of the long transfer stage between reactor outlet and expansion chamber no water has condensed and that the gas composition at the reactor outlet is retained up to the sampling point. Moreover, the good agreement with the reference measurements for all major species shows that the transfer line effect on evaluated molar fraction is negligible.

\section{E. Quantification of intermediate species at low temperature}

Intermediate species are quantified according to the principles outlined by Oßwald et al. (Oßwald et al. (2014)) for imaging PEPICO flame data using a modification of the approach for flame experiments (Schenk et al. (2013); Kasper et al. (2009)) for the evaluation of PI-MBMS data. The modification was required because of the continuous extraction of ions from the beam interaction region in contrast to pulsed or gated extraction for regular ToF-MS (Oßwald et al. (2014)) and the particularities of imaging coincidence detection. The ion signal dependency $S_{i}$ on experimental and machine parameters for data reduction of the iPEPICO instrument is elaborated by Oßwald et al. (Oßwald et al. (2014)) and has to be slightly modified for the reactor experiment to account for the difference to flame experiments:

$$
S_{i}=x_{i}(T) \cdot D_{i}\left(M_{i}\right) \cdot t \cdot F K T\left(T_{i n j}, \bar{M}\right) \cdot c(E) \cdot \sigma_{i}(E) \cdot \alpha_{i}(E-I P)
$$

Where $x_{i}$ denotes the mole fraction of a species $i$ at a set reactor temperature $T ; D$ is a mass discrimination factor dependent on mass $M$ (empirically close to unity for the CRF-PEPICO); $t$ is the 
signal accumulation time for a spectrum; FKT is the composition and temperature dependent sampling function, $T_{\text {inj }}$ is the temperature at which the sample is injected into the spectrometer, i.e., not the process temperature as $T_{\mathrm{inj}} \neq T ; c$ is a machine and experiment specific factor taking into account several machine and experiment dependent disturbances; $\sigma_{i}$ is the partial ionization cross section at the given mass-to-charge ratio; $\alpha$ corrects for loss of ion coincidence signal due to the limited electron detector kinetic energy acceptance and is required for photon energies $1.2 \mathrm{eV}$ larger than the IE of a compound (Oßwald et al. (2014)). This aspect requires quantification of a species within this $1.2 \mathrm{eV}$ boundary to obtain the most accurate result. Modifications to (1) required for the reactor experiment are the dependency of the mole fraction $x_{i}$ on the set reactor temperature $T$ instead of the height above burner for flame experiments and the changed indexing of the temperature $T_{\text {inj }}$ at which the molecular beam is formed and the sample is injected into the spectrometer.

Representative quantification results of two important intermediate species for the condition given in Table 1 are presented in Figure 7. Spectra are taken at several photon energies ranging from 8.8 $\mathrm{eV}$ to $15.8 \mathrm{eV}$ for all temperatures, considering the requirement of $\Delta E<1.2 \mathrm{eV}$ between the IE of any species of interest and the set photon energy for the quantified signal. Intermediate species are evaluated within these bounds, hence the $\alpha$ factor becomes unity.
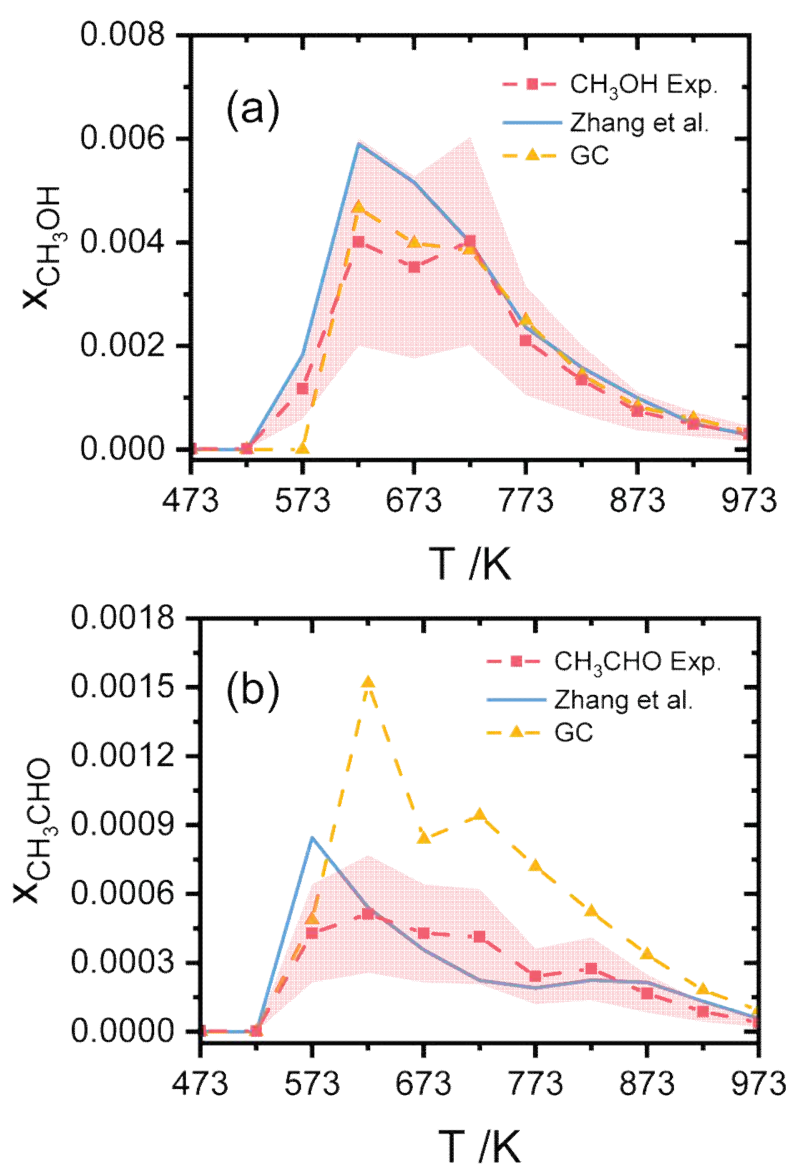

Figure 7: Quantification of low-temperature intermediate species methanol $\left(\mathrm{CH}_{3} \mathrm{OH}\right.$, Panel (a)) and acetaldehyde $\left(\mathrm{CH}_{3} \mathrm{CHO}\right.$, Panel (b)) (dashed line with symbols; red; indicated error $50 \%$ ), comparison to mechanism by Zhang et al. (Zhang et al. (2016)) (solid line; blue), and GC reference measurements cf. (Kaczmarek et al. (2019b)) (dashed line with symbols; yellow). 
Evaluation is performed for the methanol signal at a mass-to-charge ratio of $m / z 32$ (Figure 7 (a)) and the acetaldehyde signal at $m / z 44$ Figure 7 (b). The methanol signal is taken from a temperature scan performed at a photon energy of $11.2 \mathrm{eV}$, while the acetaldehyde signal was acquired at $10.6 \mathrm{eV}$. The averaging time for acquisition was $180 \mathrm{~s}$ for both species. Photoionization cross sections are taken from Cool et al. (Cool, Wang, et al. (2005); Cool et al. (2003)). For argon, the lowest energy absolute cross section available at $15.83 \mathrm{eV}$ is used (Marr \& West (1976)). The obtained mole fraction profiles are compared to the mechanism by Zhang et al. (Zhang et al. (2016)) and the GC reference data (Kaczmarek et al. (2019a)). The profiles show excellent agreement with both the measured and modeled data, as the error margin for the $\mathrm{CH}_{3} \mathrm{CHO}$ GC data is reported as factor 2-4 due to a lack of calibration (Kaczmarek et al. (2019a)). Experimental values are well within the expected $50 \%$ error margin for the PI-MBMS, indicated in Figure 7 (Oßwald et al. (2014)). This demonstrates the ability to generate quantitative speciation data for combustion experiments with the new reactor setup.

\section{CONCLUSION}

This paper describes the first utilization of a pressurized flow reactor for combustion experiments coupled to double imaging PEPICO using tunable synchrotron VUV photoionization. A detailed overview of all systems comprising the experiment, including the supply and support systems, is given. The experimental and evaluation procedures for the novel experiment are described.

Application of the setup to combustion research is demonstrated comprehensively and exemplary data are presented. The $i^{2}$ PEPICO method affords excellent identification of isomers as exemplarily shown by deconvoluting the $\mathrm{m} / \mathrm{z} 72$ to 2-butanone and $\mathrm{n}$-butanal contributions and ruling out the presence of numerous other isomers. With the technique, isomer branching ratios can be obtained, and fed back into reaction mechanism development. Identification using both the ionization potential based photoionization spectra and threshold photoelectron spectra fingerprinting is demonstrated for the two compounds. The experiment is limited to the detection of comparably stable species, which survive the expansion stages while transient radicals are lost in the sampling process. It is shown that this limitation to non-reactive species is not a decisively negative factor in the current experiment because highly reactive radicals are typically produced close to the inlet of the reactor and have been consumed in reactions close to the outlet of the reactor. In fact, the lack of radicals in the sample can be advantageous for complex samples such as the one generated by the higher hydrocarbon $\mathrm{n}$-heptane presented in this work.

Quantitative major and intermediate species profiles obtained from the pressurized reactor experiment coupled to $i^{2} P E P I C O$ are presented. They confirm the capability of the experiment to sensitively, selectively, and accurately detect key combustion species at technically relevant pressure conditions. Reliable experimental quantitative data for fuel oxidation at elevated pressure are limited and a key requisite for developing reaction mechanism and a subsequent understanding of the reactive processes. Combined with the capability to identify compounds present in the sample using threshold photoelectron spectra and ion yield curves, the new experiment presents a valuable addition to the analytical techniques employed in combustion research.

\section{ACKNOWLEDGEMENTS}

All authors thank the Deutsche Forschungsgemeinschaft for financial support under contracts KA3871/3-2, KA3871/1-3 and KO4786/2-2. P.H. also thanks the Swiss Federal Office of Energy for financial support under contract $S I / 501269-01$. Experiments were performed the VUV beamline of the SLS at Paul Scherrer Institute. We thank P. Oßwald for his support in the experiments. The authors would like to acknowledge Bálint Sztáray and David Osborn for the design of the CRF-PEPICO endstation. 
References

Ansys Inc. "Ansys Chemkin 19.2," (2008).

Baer, T., \& Tuckett, R.P. "Advances in threshold photoelectron spectroscopy (TPES) and threshold photoelectron photoion coincidence (TPEPICO)," Physical Chemistry Chemical Physics 19, 96989723 (2017).

Behan, J.M., Dean, F.M., \& Johnstone, R.A.W. "Photoelectron spectra of cyclic aromatic ethers. The question of the mills-nixon effect," Tetrahedron 32, 167-171 (1976).

Bierkandt, T., Hemberger, P., Oßwald, P., Köhler, M., \& Kasper, T. "Insights in m-xylene decomposition under fuel-rich conditions by imaging photoelectron photoion coincidence spectroscopy," Proceedings of the Combustion Institute 36, 1223-1232 (2017).

Bodi, A., Hemberger, P., Gerber, T., \& Sztáray, B. "A new double imaging velocity focusing coincidence experiment: I2PEPICO," Review of Scientific Instruments 83, (2012).

Bodi, A., Hemberger, P., Osborn, D.L., \& Sztáray, B. "Mass-resolved isomer-selective chemical analysis with imaging photoelectron photoion coincidence spectroscopy," Journal of Physical Chemistry Letters 4, 2948-2952 (2013).

Bodi, A., Johnson, M., Gerber, T., Gengeliczki, Z., Sztáray, B., \& Baer, T. "Imaging photoelectron photoion coincidence spectroscopy with velocity focusing electron optics," Review of Scientific Instruments 80, 034101 (2009).

Cool, T.A., Mcllroy, A., Qi, F., Westmoreland, P.R., Poisson, L., Peterka, D.S., \& Ahmed, M. "Photoionization mass spectrometer for studies of flame chemistry with a synchrotron light source," Review of Scientific Instruments 76, 094102 (2005).

Cool, T.A., Nakajima, K., Mostefaoui, T.A., Qi, F., Mcllroy, A., Westmoreland, P.R., Law, M.E., Poisson, L., Peterka, D.S., \& Ahmed, M. "Selective detection of isomers with photoionization mass spectrometry for studies of hydrocarbon flame chemistry," Journal of Chemical Physics 119, 8356-8365 (2003).

Cool, T.A., Wang, J., Nakajima, K., Taatjes, C.A., \& Mcllroy, A. "Photoionization cross sections for reaction intermediates in hydrocarbon combustion," International Journal of Mass Spectrometry 247, 18-27 (2005).

Curran, H.J., Gaffuri, P., Pitz, W.J., \& Westbrook, C.K. "A comprehensive modeling study of n-heptane oxidation," Combustion and Flame 114, 149-177 (1998).

Doucet, J., Sauvageau, P., \& Sandorfy, C. "The Vacuum Ultraviolet Spectrum Of Tetrahydrofuran," Chemical Physics Letters 17, 316-319 (1972).

Eskola, A.J., Antonov, I.O., Sheps, L., Savee, J.D., Osborn, D.L., \& Taatjes, C.A. "Time-resolved measurements of product formation in the low-temperature $(550-675 \mathrm{~K})$ oxidation of neopentane: A probe to investigate chain-branching mechanism," Physical Chemistry Chemical Physics 19, 13731-13745 (2017).

Felsmann, D., Lucassen, A., Krüger, J., Hemken, C., Tran, L.S., Pieper, J., Garcia, G.A., Brockhinke, A., Nahon, L., \& Kohse-Höinghaus, K. "Progress in Fixed-Photon-Energy Time-Efficient Double Imaging Photoelectron/Photoion Coincidence Measurements in Quantitative Flame Analysis," Zeitschrift fur Physikalische Chemie 230, 1067-1097 (2016). 
Felsmann, D., Moshammer, K., Krüger, J., Lackner, A., Brockhinke, A., Kasper, T., Bierkandt, T., Akyildiz, E., Hansen, N., Lucassen, A., Oßwald, P., Köhler, M., Garcia, G.A., Nahon, L., Hemberger, P., Bodi, A., Gerber, T., \& Kohse-Höinghaus, K. "Electron ionization, photoionization and photoelectron/photoion coincidence spectroscopy in mass-spectrometric investigations of a low-pressure ethylene/oxygen flame," Proceedings of the Combustion Institute 35, 779-786 (2015).

Garcia, G.A., Cunha De Miranda, B.K., Tia, M., Daly, S., \& Nahon, L. "DELICIOUS III: A multipurpose double imaging particle coincidence spectrometer for gas phase vacuum ultraviolet photodynamics studies," Review of Scientific Instruments 84, (2013).

Hansen, N., Cool, T.A., Westmoreland, P.R., \& Kohse-Höinghaus, K. "Recent contributions of flamesampling molecular-beam mass spectrometry to a fundamental understanding of combustion chemistry," Progress in Energy and Combustion Science 35, 168-191 (2009).

Hemberger, P., Trevitt, A.J., Ross, E., \& Da Silva, G. "Direct observation of para-xylylene as the decomposition product of the meta-xylyl radical using VUV synchrotron radiation," Journal of Physical Chemistry Letters 4, 2546-2550 (2013).

Herbinet, O., Battin-Leclerc, F., Bax, S., Gall, H. Le, Glaude, P.A., Fournet, R., Zhou, Z., Deng, L., Guo, H., Xie, M., \& Qi, F. "Detailed product analysis during the low temperature oxidation of nbutane," Physical Chemistry Chemical Physics 13, 296-308 (2011).

Hernandez, G.J. "Vacuum-ultraviolet absorption spectra of the cyclic ethers: Trimethylene oxide, tetrahydrofuran, and tetrahydropyran," The Journal of Chemical Physics 38, 2233-2242 (1963).

Hernandez, R., Masclet, P., \& Mouvier, G. "Spectroscopie De Photoelectron D’Aldehydes Et De Cetones Aliphatiques," Journal of Electron Spectroscopy And Related Pehnomena 10, 333-347 (1977).

Johnson, M., Bodi, A., Schulz, L., \& Gerber, T. "Vacuum ultraviolet beamline at the Swiss Light Source for chemical dynamics studies," Nuclear Instruments and Methods in Physics Research, Section A: Accelerators, Spectrometers, Detectors and Associated Equipment 610, 597-603 (2009).

Kaczmarek, D., Atakan, B., \& Kasper, T. "Investigation of the partial oxidation of methane/n-heptanemixtures and the interaction of methane and n-heptane under ultra-rich conditions," Combustion and Flame 205, 345-357 (2019)(a).

Kaczmarek, D., Atakan, B., \& Kasper, T. "Plug-Flow Reactor Study of the Partial Oxidation of Methane and Natural Gas at Ultra-Rich Conditions," Combustion Science and Technology 9, 1571-1548 (2019)(b).

Kasper, T., Oßwald, P., Struckmeier, U., Kohse-Höinghaus, K., Taatjes, C.A., Wang, J., Cool, T.A., Law, M.E., Morel, A., \& Westmoreland, P.R. "Combustion chemistry of the propanol isomers investigated by electron ionization and VUV-photoionization molecular-beam mass spectrometry," Combustion and Flame 156, 1181-1201 (2009).

Krüger, D., Oßwald, P., Köhler, M., Hemberger, P., Bierkandt, T., Karakaya, Y., \& Kasper, T. "Hydrogen abstraction ratios: A systematic iPEPICO spectroscopic investigation in laminar flames," Combustion and Flame 191, 343-352 (2018).

Krüger, D., Oßwald, P., Köhler, M., Hemberger, P., Bierkandt, T., \& Kasper, T. "The fate of the $\mathrm{OH}$ radical in molecular beam sampling experiments," Proceedings of the Combustion Institute 37, 1563-1570 (2019). 
Krüger, J., Garcia, G.A., Felsmann, D., Moshammer, K., Lackner, A., Brockhinke, A., Nahon, L., \& Kohse-Höinghaus, K. "Photoelectron-photoion coincidence spectroscopy for multiplexed detection of intermediate species in a flame," Physical Chemistry Chemical Physics 16, 2279122804 (2014).

Lindstrom, P.J., Mallard, W.G., \& National Institute of Standards and Technology (U.S.). "NIST Chemistry Webbook," (1997).

Lucassen, A., Oßwald, P., Struckmeier, U., Kohse-Höinghaus, K., Kasper, T., Hansen, N., Cool, T.A., \& Westmoreland, P.R. "Species identification in a laminar premixed low-pressure flame of morpholine as a model substance for oxygenated nitrogen-containing fuels," Proceedings of the Combustion Institute 32 I, 1269-1276 (2009).

Luck, C.J., Burgess, A.R., Desty, D.H., Whitehead, D.M., \& Pratley, G. "A study of the combustion of nheptane in an engine using a novel high-speed sampling technique," Symposium (International) On Combustion 14, 501-512 (1973).

Mackie, R.A., Scully, S.W.J., Sands, A.M., Browning, R., Dunn, K.F., \& Latimer, C.J. "A photoionization mass spectrometric study of acetylene and ethylene in the VUV spectral region," International Journal of Mass Spectrometry 223-224, 67-79 (2003).

Marr, G. V., \& West, J.B. "Absolute photoionization cross-section tables for helium, neon, argon, and krypton in the VUV spectral regions," Atomic Data and Nuclear Data Tables 18, 497-508 (1976).

Matras, D., \& Villermaux, J. "Un reakteur continu parfaitement agité par jets gazeux pour l'étude cinétique de réactions chimiques rapides," Chemical Engineering Science 28, 129-137 (1973).

Moshammer, K., Jasper, A.W., Popolan-Vaida, D.M., Lucassen, A., Diévart, P., Selim, H., Eskola, A.J., Taatjes, C.A., Leone, S.R., Sarathy, S.M., Ju, Y., Dagaut, P., Kohse-Höinghaus, K., \& Hansen, N. "Detection and Identification of the Keto-Hydroperoxide ( $\mathrm{HOOCH} 2 \mathrm{OCHO})$ and Other Intermediates during Low-Temperature Oxidation of Dimethyl Ether," Journal of Physical Chemistry A 119, 7361-7374 (2015).

Moshammer, K., Jasper, A.W., Popolan-Vaida, D.M., Wang, Z., Bhavani Shankar, V.S., Ruwe, L., Taatjes, C.A., Dagaut, P., \& Hansen, N. "Quantification of the Keto-Hydroperoxide $(\mathrm{HOOCH} 2 \mathrm{OCHO})$ and Other Elusive Intermediates during Low-Temperature Oxidation of Dimethyl Ether," Journal of Physical Chemistry A 120, 7890-7901 (2016).

Oßwald, P., Hemberger, P., Bierkandt, T., Akyildiz, E., Köhler, M., Bodi, A., Gerber, T., \& Kasper, T. "In situ flame chemistry tracing by imaging photoelectron photoion coincidence spectroscopy," Review of Scientific Instruments 85, (2014).

Oßwald, P., \& Köhler, M. "An atmospheric pressure high-temperature laminar flow reactor for investigation of combustion and related gas phase reaction systems," Review of Scientific Instruments 86, (2015).

Osswald, P., Struckmeier, U., Kasper, T., Kohse-Höinghaus, K., Wang, J., Cool, T.A., Hansen, N., \& Westmoreland, P.R. "Isomer-specific fuel destruction pathways in rich flames of methyl acetate and ethyl formate and consequences for the combustion chemistry of esters," Journal of Physical Chemistry A 111, 4093-4101 (2007).

Pelucchi, M., Bissoli, M., Cavallotti, C., Cuoci, A., Faravelli, T., Frassoldati, A., Ranzi, E., \& Stagni, A. "Improved kinetic model of the low-temperature oxidation of $n$-heptane," Energy and Fuels 28 , 7178-7193 (2014). 
Person, J.C., \& Nicole, P.P. "Isotope Effects in the Photoionization Yields and in the Absorption Cross Sections for Methanol, Ethanol, Methyl Bromide, and Ethyl Bromide," The Journal of Chemical Physics 55, 3390-3397 (1971).

Pieper, J., Schmitt, S., Hemken, C., Davies, E., Wullenkord, J., Brockhinke, A., Krüger, J., Garcia, G.A., Nahon, L., Lucassen, A., Eisfeld, W., \& Kohse-Höinghaus, K. "Isomer Identification in Flames with Double-Imaging Photoelectron/Photoion Coincidence Spectroscopy (i2PEPICO) using Measured and Calculated Reference Photoelectron Spectra," Zeitschrift fur Physikalische Chemie 232, 153-187 (2018).

Qi, F. "Combustion chemistry probed by synchrotron VUV photoionization mass spectrometry," Proceedings of the Combustion Institute 34, 33-63 (2013).

Ranzi, E., Gaffuri, P., Faravelli, T., \& Dagaut, P. "A wide-range modeling study of n-heptane oxidation," Combustion and Flame 103, 91-106 (1995).

Rasmussen, C.L., Hansen, J., Paul, M., \& Glarborg, P. "Experimental measurements and kinetic modeling of $\mathrm{CO} / \mathrm{H} 2 / \mathrm{O} 2 / \mathrm{NOx}$ conversion at high pressure," International Journal of Chemical Kinetics 40, 454-480 (2008).

Schenk, M., Leon, L., Moshammer, K., Oßwald, P., Zeuch, T., Seidel, L., Mauss, F., \& Kohse-Höinghaus, K. "Detailed mass spectrometric and modeling study of isomeric butene flames," Combustion and Flame 160, 487-503 (2013).

Sztáray, B., Voronova, K., Torma, K.G., Covert, K.J., Bodi, A., Hemberger, P., Gerber, T., \& Osborn, D.L. "CRF-PEPICO: Double velocity map imaging photoelectron photoion coincidence spectroscopy for reaction kinetics studies," Journal of Chemical Physics 147, (2017).

Traeger, J.C. "Heat of formation for the SH radical by photoionization mass spectrometry," Organic Mass Spectrometry 19, 514-516 (1984).

Traeger, J.C., \& McAdoo, D.J. "Decomposition thresholds and associated translational energy releases for eight $\mathrm{C} 4 \mathrm{H} 8 \mathrm{O}+$. isomers," International Journal of Mass Spectrometry and Ion Processes 68, 35-48 (1986).

Watanabe, K., Nakayama, T., \& Mottl, J. "lonization potentials of some molecules," Journal of Quantitative Spectroscopy and Radiative Transfer 2, 369-382 (1962).

Wiley, W.C., \& McLaren, I.H. "Time-of-flight mass spectrometer with improved resolution," Review of Scientific Instruments 26, 1150-1157 (1955).

Zhang, K., Banyon, C., Bugler, J., Curran, H.J., Rodriguez, A., Herbinet, O., Battin-Leclerc, F., B'Chir, C., \& Heufer, K.A. "An updated experimental and kinetic modeling study of $n$-heptane oxidation," Combustion and Flame 172, 116-135 (2016).

Zhou, Z., Xie, M., Wang, Z., \& Qi, F. "Determination of absolute photoionization cross-sections of aromatics and aromatic derivatives," Rapid Communications in Mass Spectrometry 23, 39944002 (2009). 